Prepared for the U.S. Department of Energy

under Contract DE-AC05-76RL01830

\title{
New Stochastic Annual Limits on Intake for Selected Radionuclides
}

\author{
EH Carbaugh
}

August 2009

Pacific Northwest NATIONAL LABORATORY

Proudly Operated by Battelle Since 1965 


\title{
DISCLAIMER
}

This report was prepared as an account of work sponsored by an agency of the United States Government. Neither the United States Government nor any agency thereof, nor Battelle Memorial Institute, nor any of their employees, makes any warranty, express or implied, or assumes any legal liability or responsibility for the accuracy, completeness, or usefulness of any information, apparatus, product, or process disclosed, or represents that its use would not infringe privately owned rights. Reference herein to any specific commercial product, process, or service by trade name, trademark, manufacturer, or otherwise does not necessarily constitute or imply its endorsement, recommendation, or favoring by the United States Government or any agency thereof, or Battelle Memorial Institute. The views and opinions of authors expressed herein do not necessarily state or reflect those of the United States Government or any agency thereof.

\author{
PACIFIC NORTHWEST NATIONAL LABORATORY \\ operated by \\ BATTELLE \\ for the \\ UNITED STATES DEPARTMENT OF ENERGY \\ under Contract DE-AC05-76RLO1830
}

Printed in the United States of America
Available to DOE and DOE contractors from the Office of Scientific and Technical Information,
P.O. Box 62, Oak Ridge, TN 37831-0062;
ph: (865) 576-8401
fax: $(865)$ 576-5728
email: reports@adonis.osti.gov

\author{
Available to the public from the National Technical Information Service, \\ U.S. Department of Commerce, 5285 Port Royal Rd., Springfield, VA 22161 \\ ph: (800) 553-6847 \\ fax: (703) 605-6900 \\ email: orders $@$ ntis.fedworld.gov \\ online ordering: http://www.ntis.gov/ordering.htm
}

This document was printed on recycled paper.

$(9 / 2003)$ 


\section{New Stochastic Annual Limits on Intake for Selected Radionuclides}

EH Carbaugh

August 2009

Prepared for

the U.S. Department of Energy

under Contract DE-AC05-76RL01830

Pacific Northwest National Laboratory

Richland, Washington 99352 
PNNL-18690

\section{Contents}

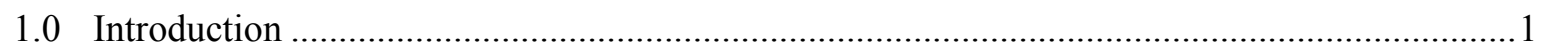

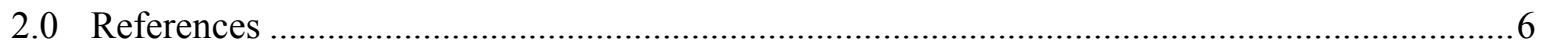




\subsection{Introduction}

Annual limits on intake (ALI) have historically been tabulated by the International Commission on Radiological Protection (e.g., ICRP 1979, 1961) and also by the Environmental Protection Agency (EPA 1988). These compilations have been rendered obsolete by more recent ICRP dosimetry methods, and, rather than provide new ALIs, the ICRP has opted instead to provide committed dose coefficients from which an ALI can be determined by a user for a specific set of conditions. The U.S. Department of Energy historically has referenced compilations of ALIs and has defined their method of calculation in its radiation protection regulation (10 CFR 835), but has never provided a specific compilation. Under June 2007 amendments to 10 CFR 835, ALIs can be calculated by dividing an appropriate dose limit, either 5rem $(0.05 \mathrm{~Sv})$ effective dose or $50 \mathrm{rem}(0.5 \mathrm{~Sv})$ equivalent dose to an individual organ or tissue, by an appropriate committed dose coefficient. When based on effective dose, the ALI is often referred to as a stochastic annual limit on intake (SALI), and when based on the individual organ or tissue equivalent limit, it has often been called a deterministic annual limit on intake (DALI).

This report compiles committed effective dose coefficients and SALIs for inhalation of $5-\mu \mathrm{m}$ activity median aerodynamic diameter (AMAD) particles. These SALIs are compatible with the June 2007 amendments to 10 CFR 835, and were derived using the dose coefficients of the ICRP Database of Dose Coefficients (ICRP 1998), and the 5-rem (0.05 Sv) effective dose limit of 10 CFR 835 according to the following relationship:

$$
S A L I(B q)=\frac{0.05 S v}{e(50)}
$$

where $\mathrm{e}(50)$ is the committed effective dose coefficient in units of Sv Bq-1.

The entries for H-3 Type F, M, and S metal tritides were based on dose coefficients published in Appendix A of the "DOE Handbook Radiological Control Programs for Special Tritum Compounds (DOE 2004).

The SALI activities were converted from the International System of Units (SI) unit of becquerel $(\mathrm{Bq})$ to the conventional unit of microcurie $(\mu \mathrm{Ci}$ ) by dividing by 37,000 , based on the fundamental relationship of $1 \mu \mathrm{Ci}$ equaling $37,000 \mathrm{~Bq}$.

The SALIs compiled in this report are for radionuclides identified by the Pacific Northwest National Laboratory and Hanford Site contractors as pertinent or potentially pertinent to radiation work permits associated with various waste management and research activities. The SALIs are not necessarily the most limiting of the annual limits on intake, however they are useful for determining if bioassay monitoring may be warranted based on the amount of radioactive material in process. Bioassay monitoring is required by 10 CFR 835.402(c)(1) if a worker is likely to incur an intake resulting in a committed effective dose of $100 \mathrm{mrem}$, which corresponds to an intake of $2 \%$ of a SALI. This compilation of SALIs is intended as a convenient reference for SALI values which might be used for such determinations. There is no intent for this compilation to be a comprehensive list. 
Table 1. (contd)

Table 1. Selected Effective Dose Coefficients and Stochastic Annual Limits on Intake

\begin{tabular}{|c|c|c|c|c|c|}
\hline \multirow[b]{2}{*}{ Radionuclide } & \multirow{2}{*}{$\begin{array}{c}\text { Absorption } \\
\text { Type }\end{array}$} & \multirow{2}{*}{$\begin{array}{l}5-\mu \mathrm{m} \text { Inhalation } \\
\mathrm{e}(50) \text { in } \mathrm{Sv} \mathrm{Bq}-1\end{array}$} & \multicolumn{2}{|c|}{ Stochastic ALI } & \\
\hline & & & $(\mathrm{Bq})$ & $(\mu \mathrm{Ci})$ & \\
\hline Ac-225 & $\mathrm{F}$ & $1.0 \mathrm{E}-06$ & $5.0 \mathrm{E}+04$ & $1.4 \mathrm{E}+00$ & \\
\hline Ac- 225 & $\mathrm{M}$ & $5.7 \mathrm{E}-06$ & $8.8 \mathrm{E}+03$ & $2.4 \mathrm{E}-01$ & \\
\hline Ac- 225 & $\mathrm{~S}$ & $6.5 \mathrm{E}-06$ & $7.7 \mathrm{E}+03$ & $2.1 \mathrm{E}-01$ & \\
\hline Ac- -227 & $\mathrm{~F}$ & $6.3 \mathrm{E}-04$ & $7.9 \mathrm{E}+01$ & $2.1 \mathrm{E}-03$ & \\
\hline Ac-227 & M & $1.5 \mathrm{E}-04$ & $3.3 \mathrm{E}+02$ & $9.0 \mathrm{E}-03$ & \\
\hline Ac- 227 & $\mathrm{~S}$ & 4.7E-05 & $1.1 \mathrm{E}+03$ & $2.9 \mathrm{E}-02$ & \\
\hline Ac- -228 & $\mathrm{~F}$ & 2.9E-08 & $1.7 \mathrm{E}+06$ & $4.7 \mathrm{E}+01$ & \\
\hline Ac- 228 & $M \& S$ & $1.2 \mathrm{E}-08$ & $4.2 \mathrm{E}+06$ & $1.1 \mathrm{E}+02$ & \\
\hline Ag-108m & $\mathrm{F}$ & 7.3E-09 & $6.8 \mathrm{E}+06$ & $1.9 \mathrm{E}+02$ & \\
\hline $\mathrm{Ag}-110 \mathrm{~m}$ & $\mathrm{~S}$ & 7.3E-09 & $6.8 \mathrm{E}+06$ & $1.9 \mathrm{E}+02$ & \\
\hline Ag-111 & $\mathrm{S}$ & $1.6 \mathrm{E}-09$ & $3.1 \mathrm{E}+07$ & $8.4 \mathrm{E}+02$ & \\
\hline Al-26 & $\mathrm{F}$ & $1.4 \mathrm{E}-08$ & $3.6 \mathrm{E}+06$ & $9.7 \mathrm{E}+01$ & \\
\hline Am-241 & M & $2.7 \mathrm{E}-05$ & $1.9 \mathrm{E}+03$ & $5.0 \mathrm{E}-02$ & \\
\hline Am-242m & $\mathrm{M}$ & $2.4 \mathrm{E}-05$ & $2.1 \mathrm{E}+03$ & $5.6 \mathrm{E}-02$ & \\
\hline Am-243 & M & $2.7 \mathrm{E}-05$ & $1.9 \mathrm{E}+03$ & $5.0 \mathrm{E}-02$ & \\
\hline Am-244 & M & $1.5 \mathrm{E}-09$ & $3.3 \mathrm{E}+07$ & $9.0 \mathrm{E}+02$ & \\
\hline As-76 & $\mathrm{M}$ & $9.2 \mathrm{E}-10$ & $5.4 \mathrm{E}+07$ & $1.5 \mathrm{E}+03$ & \\
\hline $\mathrm{Au}-195$ & $\mathrm{~S}$ & $1.2 \mathrm{E}-09$ & $4.2 \mathrm{E}+07$ & $1.1 \mathrm{E}+03$ & \\
\hline $\mathrm{Au}-198$ & $\mathrm{~S}$ & $1.1 \mathrm{E}-09$ & $4.5 \mathrm{E}+07$ & $1.2 \mathrm{E}+03$ & \\
\hline Ba-131 & $\mathrm{F}$ & $3.5 \mathrm{E}-10$ & $1.4 \mathrm{E}+08$ & $3.9 \mathrm{E}+03$ & \\
\hline Ba-133 & $\mathrm{F}$ & $1.8 \mathrm{E}-09$ & $2.8 \mathrm{E}+07$ & $7.5 \mathrm{E}+02$ & \\
\hline Ba-140 & $\mathrm{F}$ & $1.6 \mathrm{E}-09$ & $3.1 \mathrm{E}+07$ & $8.4 \mathrm{E}+02$ & \\
\hline Be-10 & $\mathrm{S}$ & $1.9 \mathrm{E}-08$ & $2.6 \mathrm{E}+06$ & $7.1 \mathrm{E}+01$ & \\
\hline $\mathrm{Be}-7$ & $\mathrm{~S}$ & $4.6 \mathrm{E}-11$ & $1.1 \mathrm{E}+09$ & $2.9 \mathrm{E}+04$ & \\
\hline Bi-207 & M & $3.2 \mathrm{E}-09$ & $1.6 \mathrm{E}+07$ & $4.2 \mathrm{E}+02$ & \\
\hline $\mathrm{Bi}-210$ & M & $6.0 \mathrm{E}-08$ & $8.3 \mathrm{E}+05$ & $2.3 \mathrm{E}+01$ & \\
\hline Bi-212 & M & $3.9 \mathrm{E}-08$ & $1.3 \mathrm{E}+06$ & $3.5 \mathrm{E}+01$ & \\
\hline $\mathrm{Bi}-214$ & M & $2.1 \mathrm{E}-08$ & $2.4 \mathrm{E}+06$ & $6.4 \mathrm{E}+01$ & \\
\hline Bk-249 & M & $1.0 \mathrm{E}-07$ & $5.0 \mathrm{E}+05$ & $1.4 \mathrm{E}+01$ & \\
\hline $\mathrm{Br}-82$ & M & $8.8 \mathrm{E}-10$ & $5.7 \mathrm{E}+07$ & $1.5 \mathrm{E}+03$ & \\
\hline $\mathrm{C}-14$ & $*$ & $5.8 \mathrm{E}-10$ & $8.6 \mathrm{E}+07$ & $2.3 \mathrm{E}+03$ & * organic gases and vapors \\
\hline $\mathrm{Ca}-45$ & M & 2.3E-09 & $2.2 \mathrm{E}+07$ & $5.9 \mathrm{E}+02$ & \\
\hline Cd-109 & $\mathrm{F}$ & $9.6 \mathrm{E}-09$ & $5.2 \mathrm{E}+06$ & $1.4 \mathrm{E}+02$ & \\
\hline $\mathrm{Cd}-113 \mathrm{~m}$ & $\mathrm{~F}$ & $1.3 \mathrm{E}-07$ & $3.8 \mathrm{E}+05$ & $1.0 \mathrm{E}+01$ & \\
\hline Ce-139 & $\mathrm{S}$ & $1.4 \mathrm{E}-09$ & $3.6 \mathrm{E}+07$ & $9.7 \mathrm{E}+02$ & \\
\hline $\mathrm{Ce}-144$ & $\mathrm{~S}$ & 2.9E-08 & $1.7 \mathrm{E}+06$ & $4.7 \mathrm{E}+01$ & \\
\hline Cf-252 & M & $1.3 \mathrm{E}-05$ & $3.8 \mathrm{E}+03$ & $1.0 \mathrm{E}-01$ & \\
\hline $\mathrm{Cl}-36$ & M & $5.1 \mathrm{E}-09$ & $9.8 \mathrm{E}+06$ & $2.6 \mathrm{E}+02$ & \\
\hline $\mathrm{Cm}-242$ & $\mathrm{M}$ & $3.7 \mathrm{E}-06$ & $1.4 \mathrm{E}+04$ & $3.7 \mathrm{E}-01$ & \\
\hline $\mathrm{Cm}-243$ & $\mathrm{M}$ & $2.0 \mathrm{E}-05$ & $2.5 \mathrm{E}+03$ & $6.8 \mathrm{E}-02$ & \\
\hline $\mathrm{Cm}-244$ & M & $1.7 \mathrm{E}-05$ & $2.9 \mathrm{E}+03$ & 7.9E-02 & \\
\hline $\mathrm{Cm}-248$ & $\mathrm{M}$ & $9.5 \mathrm{E}-05$ & $5.3 \mathrm{E}+02$ & $1.4 \mathrm{E}-02$ & \\
\hline Co-57 & $\mathrm{M}$ & $3.9 \mathrm{E}-10$ & $1.3 \mathrm{E}+08$ & $3.5 \mathrm{E}+03$ & \\
\hline Co-57 & $\mathrm{S}$ & $6.0 \mathrm{E}-10$ & $8.3 \mathrm{E}+07$ & $2.3 \mathrm{E}+03$ & \\
\hline
\end{tabular}


Table 1. (contd)

\begin{tabular}{|c|c|c|c|c|c|}
\hline \multirow[b]{2}{*}{ Radionuclide } & \multirow{2}{*}{$\begin{array}{c}\text { Absorption } \\
\text { Type }\end{array}$} & \multirow{2}{*}{$\begin{array}{l}\text { 5- } \mu \mathrm{m} \text { Inhalation } \\
\mathrm{e}(50) \text { in } \mathrm{Sv} \mathrm{Bq}-1\end{array}$} & \multicolumn{2}{|c|}{ Stochastic ALI } & \\
\hline & & & $(\mathrm{Bq})$ & $(\mu \mathrm{Ci})$ & \\
\hline Co-58 & $\mathrm{M}$ & $1.4 \mathrm{E}-09$ & $3.6 \mathrm{E}+07$ & $9.7 \mathrm{E}+02$ & \\
\hline Co-58 & $\mathrm{S}$ & $1.7 \mathrm{E}-09$ & $2.9 \mathrm{E}+07$ & $7.9 \mathrm{E}+02$ & \\
\hline Co-60 & M & 7.1E-09 & $7.0 \mathrm{E}+06$ & $1.9 \mathrm{E}+02$ & \\
\hline Co-60 & $\mathrm{S}$ & $1.7 \mathrm{E}-08$ & $2.9 \mathrm{E}+06$ & $7.9 \mathrm{E}+01$ & \\
\hline $\mathrm{Co}-60 \mathrm{~m}$ & $M \& S$ & $1.2 \mathrm{E}-12$ & $4.2 \mathrm{E}+10$ & $1.1 \mathrm{E}+06$ & \\
\hline $\mathrm{Cr}-51$ & $\mathrm{~S}$ & $3.6 \mathrm{E}-11$ & $1.4 \mathrm{E}+09$ & $3.8 \mathrm{E}+04$ & \\
\hline Cs-131 & $\mathrm{F}$ & $4.5 \mathrm{E}-11$ & $1.1 \mathrm{E}+09$ & $3.0 \mathrm{E}+04$ & \\
\hline Cs-132 & $\mathrm{F}$ & $3.8 \mathrm{E}-10$ & $1.3 \mathrm{E}+08$ & $3.6 \mathrm{E}+03$ & \\
\hline Cs-134 & $\mathrm{F}$ & $9.6 \mathrm{E}-09$ & $5.2 \mathrm{E}+06$ & $1.4 \mathrm{E}+02$ & \\
\hline Cs-135 & $\mathrm{F}$ & $9.9 \mathrm{E}-10$ & $5.1 \mathrm{E}+07$ & $1.4 \mathrm{E}+03$ & \\
\hline Cs-136 & $\mathrm{F}$ & 1.9E-09 & $2.6 \mathrm{E}+07$ & $7.1 \mathrm{E}+02$ & \\
\hline Cs-137 & $\mathrm{F}$ & $6.7 \mathrm{E}-09$ & $7.5 \mathrm{E}+06$ & $2.0 \mathrm{E}+02$ & \\
\hline $\mathrm{Cu}-64$ & $M \& S$ & $1.5 \mathrm{E}-10$ & $3.3 \mathrm{E}+08$ & $9.0 \mathrm{E}+03$ & \\
\hline Es-253 & M & $2.1 \mathrm{E}-06$ & $2.4 \mathrm{E}+04$ & $6.4 \mathrm{E}-01$ & \\
\hline Eu-152 & M & 2.7E-08 & $1.9 \mathrm{E}+06$ & $5.0 \mathrm{E}+01$ & \\
\hline Eu-154 & $\mathrm{M}$ & $3.5 \mathrm{E}-08$ & $1.4 \mathrm{E}+06$ & $3.9 \mathrm{E}+01$ & \\
\hline Eu-155 & $\mathrm{M}$ & 4.7E-09 & $1.1 \mathrm{E}+07$ & $2.9 \mathrm{E}+02$ & \\
\hline F-18 & $\mathrm{S}$ & $9.3 \mathrm{E}-11$ & $5.4 \mathrm{E}+08$ & $1.5 \mathrm{E}+04$ & \\
\hline $\mathrm{Fe}-55$ & $\mathrm{~F}$ & $9.2 \mathrm{E}-10$ & $5.4 \mathrm{E}+07$ & $1.5 \mathrm{E}+03$ & \\
\hline $\mathrm{Fe}-59$ & $\mathrm{M}$ & $3.2 \mathrm{E}-09$ & $1.6 \mathrm{E}+07$ & $4.2 \mathrm{E}+02$ & \\
\hline Ga-72 & M & $8.4 \mathrm{E}-10$ & $6.0 \mathrm{E}+07$ & $1.6 \mathrm{E}+03$ & \\
\hline Ge-71 & M & $1.1 \mathrm{E}-11$ & $4.5 \mathrm{E}+09$ & $1.2 \mathrm{E}+05$ & \\
\hline Gd-153 & $\mathrm{F}$ & $2.5 \mathrm{E}-09$ & $2.0 \mathrm{E}+07$ & $5.4 \mathrm{E}+02$ & \\
\hline $\mathrm{H}-3$ & HTO* & $1.8 \mathrm{E}-11$ & $2.8 \mathrm{E}+09$ & $7.5 \mathrm{E}+04$ & *tritiated water/vapor \\
\hline $\mathrm{H}-3$ & $\mathrm{OBT}^{*}$ & $4.1 \mathrm{E}-11$ & $1.2 \mathrm{E}+09$ & $3.3 \mathrm{E}+04$ & * organically bound tritium \\
\hline $\mathrm{H}-3$ & Type F & & & & Based on DOE (2004 \\
\hline & tritides & $5.5 \mathrm{E}-11$ & $9.1 \mathrm{E}+08$ & $2.5 \mathrm{E}+04$ & Appendix A \\
\hline H-3 & Type M & & & & Based on DOE (2004 \\
\hline & tritides & $8.6 \mathrm{E}-11$ & $5.8 \mathrm{E}+08$ & $1.6 \mathrm{E}+04$ & Appendix A \\
\hline $\mathrm{H}-3$ & Type S & & & & Based on DOE (2004 \\
\hline & tritides & $2.6 \mathrm{E}-10$ & $1.9 \mathrm{E}+08$ & $5.2 \mathrm{E}+03$ & Appendix A \\
\hline $\mathrm{Hg}-203$ & Vapor & 7.0E-09 & $7.1 \mathrm{E}+06$ & $1.9 \mathrm{E}+02$ & \\
\hline $\mathrm{Hg}-203$ & $\mathrm{M}$ & $1.9 \mathrm{E}-09$ & $2.6 \mathrm{E}+07$ & $7.1 \mathrm{E}+02$ & \\
\hline $\mathrm{I}-125$ & Vapor & $1.4 \mathrm{E}-08$ & $3.6 \mathrm{E}+06$ & $9.7 \mathrm{E}+01$ & \\
\hline $\mathrm{I}-129$ & Vapor & $9.6 \mathrm{E}-08$ & $5.2 \mathrm{E}+05$ & $1.4 \mathrm{E}+01$ & \\
\hline $\mathrm{I}-131$ & Vapor & $2.0 \mathrm{E}-08$ & $2.5 \mathrm{E}+06$ & $6.8 \mathrm{E}+01$ & \\
\hline $\mathrm{I}-132$ & Vapor & $3.1 \mathrm{E}-10$ & $1.6 \mathrm{E}+08$ & $4.4 \mathrm{E}+03$ & \\
\hline In-111 & $\mathrm{M}$ & $3.1 \mathrm{E}-10$ & $1.6 \mathrm{E}+08$ & $4.4 \mathrm{E}+03$ & \\
\hline In- $115 \mathrm{~m}$ & M & $8.7 \mathrm{E}-11$ & $5.7 \mathrm{E}+08$ & $1.6 \mathrm{E}+04$ & \\
\hline In-116m & $\mathrm{M}$ & $8.0 \mathrm{E}-11$ & $6.3 \mathrm{E}+08$ & $1.7 \mathrm{E}+04$ & \\
\hline Ir-192 & $\mathrm{S}$ & 4.9E-09 & $1.0 \mathrm{E}+07$ & $2.8 \mathrm{E}+02$ & \\
\hline $\mathrm{K}-40$ & $\mathrm{~F}$ & $3.0 \mathrm{E}-09$ & $1.7 \mathrm{E}+07$ & $4.5 \mathrm{E}+02$ & \\
\hline La-140 & M & $1.5 \mathrm{E}-09$ & $3.3 \mathrm{E}+07$ & $9.0 \mathrm{E}+02$ & \\
\hline Mn-53 & $F \& M$ & $3.6 \mathrm{E}-11$ & $1.4 \mathrm{E}+09$ & $3.8 \mathrm{E}+04$ & \\
\hline Mn-54 & M & $1.2 \mathrm{E}-09$ & $4.2 \mathrm{E}+07$ & $1.1 \mathrm{E}+03$ & \\
\hline Mo-93 & $\mathrm{F}$ & $1.4 \mathrm{E}-09$ & $3.6 \mathrm{E}+07$ & $9.7 \mathrm{E}+02$ & \\
\hline Mo-99 & S & $1.1 \mathrm{E}-09$ & $4.5 \mathrm{E}+07$ & $1.2 \mathrm{E}+03$ & \\
\hline $\mathrm{Na}-22$ & $\mathrm{~F}$ & $2.0 \mathrm{E}-09$ & $2.5 \mathrm{E}+07$ & $6.8 \mathrm{E}+02$ & \\
\hline
\end{tabular}


Table 1. (contd)

\begin{tabular}{|c|c|c|c|c|}
\hline \multirow[b]{2}{*}{ Radionuclide } & \multirow{2}{*}{$\begin{array}{c}\text { Absorption } \\
\text { Type }\end{array}$} & \multirow{2}{*}{$\begin{array}{l}\text { 5- } \mu \mathrm{m} \text { Inhalation } \\
\mathrm{e}(50) \text { in } \mathrm{Sv} \mathrm{Bq}-1\end{array}$} & \multicolumn{2}{|c|}{ Stochastic ALI } \\
\hline & & & $(\mathrm{Bq})$ & $(\mu \mathrm{Ci})$ \\
\hline $\mathrm{Na}-24$ & $\mathrm{~F}$ & $5.3 \mathrm{E}-10$ & $9.4 \mathrm{E}+07$ & $2.5 \mathrm{E}+03$ \\
\hline $\mathrm{Nb}-93 \mathrm{~m}$ & $\mathrm{~S}$ & $8.6 \mathrm{E}-10$ & $5.8 \mathrm{E}+07$ & $1.6 \mathrm{E}+03$ \\
\hline $\mathrm{Nb}-94$ & $\mathrm{~S}$ & $2.5 \mathrm{E}-08$ & $2.0 \mathrm{E}+06$ & $5.4 \mathrm{E}+01$ \\
\hline $\mathrm{Nb}-95$ & $M \& S$ & $1.3 \mathrm{E}-09$ & $3.8 \mathrm{E}+07$ & $1.0 \mathrm{E}+03$ \\
\hline $\mathrm{Ni}-59$ & Carbonyl & $8.3 \mathrm{E}-10$ & $6.0 \mathrm{E}+07$ & $1.6 \mathrm{E}+03$ \\
\hline Ni-63 & Carbonyl & 2.0E-09 & $2.5 \mathrm{E}+07$ & $6.8 \mathrm{E}+02$ \\
\hline $\mathrm{Np}-235$ & M & $2.7 \mathrm{E}-10$ & $1.9 \mathrm{E}+08$ & $5.0 \mathrm{E}+03$ \\
\hline $\mathrm{Np}-236$ & M & $2.0 \mathrm{E}-06$ & $2.5 \mathrm{E}+04$ & $6.8 \mathrm{E}-01$ \\
\hline $\mathrm{Np}-237$ & $\mathrm{M}$ & $1.5 \mathrm{E}-05$ & $3.3 \mathrm{E}+03$ & $9.0 \mathrm{E}-02$ \\
\hline $\mathrm{Np}-239$ & $\mathrm{M}$ & $1.1 \mathrm{E}-09$ & $4.5 \mathrm{E}+07$ & $1.2 \mathrm{E}+03$ \\
\hline $\mathrm{P}-32$ & M & 2.9E-09 & $1.7 \mathrm{E}+07$ & $4.7 \mathrm{E}+02$ \\
\hline P-33 & M & $1.3 \mathrm{E}-09$ & $3.8 \mathrm{E}+07$ & $1.0 \mathrm{E}+03$ \\
\hline $\mathrm{Pb}-210$ & $\mathrm{~F}$ & $1.1 \mathrm{E}-06$ & $4.5 \mathrm{E}+04$ & $1.2 \mathrm{E}+00$ \\
\hline $\mathrm{Pb}-211$ & $\mathrm{~F}$ & $5.6 \mathrm{E}-09$ & $8.9 E+06$ & $2.4 \mathrm{E}+02$ \\
\hline $\mathrm{Pb}-212$ & $\mathrm{~F}$ & 3.3E-08 & $1.5 \mathrm{E}+06$ & $4.1 \mathrm{E}+01$ \\
\hline $\mathrm{Pb}-214$ & $\mathrm{~F}$ & 4.8E-09 & $1.0 \mathrm{E}+07$ & $2.8 \mathrm{E}+02$ \\
\hline Pd-103 & $\mathrm{M}$ & $3.0 \mathrm{E}-10$ & $1.7 \mathrm{E}+08$ & $4.5 \mathrm{E}+03$ \\
\hline Pd-107 & $\mathrm{S}$ & $2.9 \mathrm{E}-10$ & $1.7 \mathrm{E}+08$ & $4.7 \mathrm{E}+03$ \\
\hline Pm-147 & $\mathrm{M}$ & $3.5 \mathrm{E}-09$ & $1.4 \mathrm{E}+07$ & $3.9 \mathrm{E}+02$ \\
\hline Po-210 & $\mathrm{M}$ & $2.2 \mathrm{E}-06$ & $2.3 \mathrm{E}+04$ & $6.1 \mathrm{E}-01$ \\
\hline Pr-144 & $\mathrm{S}$ & $3.0 \mathrm{E}-11$ & $1.7 \mathrm{E}+09$ & $4.5 \mathrm{E}+04$ \\
\hline $\mathrm{Pu}-236$ & M & $1.3 \mathrm{E}-05$ & $3.8 \mathrm{E}+03$ & $1.0 \mathrm{E}-01$ \\
\hline $\mathrm{Pu}-237$ & $\mathrm{~S}$ & $3.0 \mathrm{E}-10$ & $1.7 \mathrm{E}+08$ & $4.5 \mathrm{E}+03$ \\
\hline $\mathrm{Pu}-238$ & M & $3.0 \mathrm{E}-05$ & $1.7 \mathrm{E}+03$ & $4.5 \mathrm{E}-02$ \\
\hline $\mathrm{Pu}-238$ & $\mathrm{~S}$ & $1.1 \mathrm{E}-05$ & $4.5 \mathrm{E}+03$ & $1.2 \mathrm{E}-01$ \\
\hline Pu-239 & $\mathrm{M}$ & $3.2 \mathrm{E}-05$ & $1.6 \mathrm{E}+03$ & 4.2E-02 \\
\hline Pu239 & $\mathrm{S}$ & 8.3E-06 & $6.0 \mathrm{E}+03$ & $1.6 \mathrm{E}-01$ \\
\hline $\mathrm{Pu}-240$ & M & $3.2 \mathrm{E}-05$ & $1.6 \mathrm{E}+03$ & 4.2E-02 \\
\hline $\mathrm{Pu}-240$ & $\mathrm{~S}$ & 8.3E-06 & $6.0 \mathrm{E}+03$ & $1.6 \mathrm{E}-01$ \\
\hline $\mathrm{Pu}-241$ & $\mathrm{M}$ & $5.8 \mathrm{E}-07$ & $8.6 \mathrm{E}+04$ & $2.3 \mathrm{E}+00$ \\
\hline $\mathrm{Pu}-241$ & $\mathrm{~S}$ & 8.4E-08 & $6.0 \mathrm{E}+05$ & $1.6 \mathrm{E}+01$ \\
\hline $\mathrm{Pu}-242$ & $\mathrm{M}$ & $3.1 \mathrm{E}-05$ & $1.6 \mathrm{E}+03$ & 4.4E-02 \\
\hline $\mathrm{Pu}-242$ & $\mathrm{~S}$ & 7.7E-06 & $6.5 \mathrm{E}+03$ & 1.8E-01 \\
\hline $\mathrm{Pu}-244$ & $\mathrm{M}$ & $3.0 \mathrm{E}-05$ & $1.7 \mathrm{E}+03$ & $4.5 \mathrm{E}-02$ \\
\hline $\mathrm{Pu}-244$ & $\mathrm{~S}$ & 7.4E-06 & $6.8 \mathrm{E}+03$ & $1.8 \mathrm{E}-01$ \\
\hline Ra-223 & M & 5.7E-06 & $8.8 \mathrm{E}+03$ & 2.4E-01 \\
\hline Ra-224 & $\mathrm{M}$ & $2.4 \mathrm{E}-06$ & $2.1 \mathrm{E}+04$ & 5.6E-01 \\
\hline Ra-225 & M & $4.8 \mathrm{E}-06$ & $1.0 \mathrm{E}+04$ & 2.8E-01 \\
\hline Ra-226 & M & $2.2 \mathrm{E}-06$ & $2.3 \mathrm{E}+04$ & $6.1 \mathrm{E}-01$ \\
\hline Ra-228 & $\mathrm{M}$ & $1.7 \mathrm{E}-06$ & $2.9 \mathrm{E}+04$ & 7.9E-01 \\
\hline $\mathrm{Rb}-86$ & $\mathrm{~F}$ & $1.3 \mathrm{E}-09$ & $3.8 \mathrm{E}+07$ & $1.0 \mathrm{E}+03$ \\
\hline $\mathrm{Rh}-103 \mathrm{~m}$ & $\mathrm{~S}$ & $2.5 \mathrm{E}-12$ & $2.0 \mathrm{E}+10$ & $5.4 \mathrm{E}+05$ \\
\hline $\mathrm{Rh}-106 \mathrm{~m}$ & $\mathrm{~S}$ & $1.9 \mathrm{E}-10$ & $2.6 \mathrm{E}+08$ & $7.1 \mathrm{E}+03$ \\
\hline $\mathrm{Ru}-103$ & $\mathrm{~S}$ & 2.2E-09 & $2.3 \mathrm{E}+07$ & $6.1 \mathrm{E}+02$ \\
\hline Ru-106 & $\mathrm{S}$ & $3.5 \mathrm{E}-08$ & $1.4 \mathrm{E}+06$ & $3.9 \mathrm{E}+01$ \\
\hline S-35 & $\mathrm{M}$ & $1.1 \mathrm{E}-09$ & $4.5 \mathrm{E}+07$ & $1.2 \mathrm{E}+03$ \\
\hline Sm-153 & $\mathrm{M}$ & $6.8 \mathrm{E}-10$ & $7.4 \mathrm{E}+07$ & $2.0 \mathrm{E}+03$ \\
\hline $\mathrm{Sb}-125$ & $\mathrm{M}$ & 3.3E-09 & $1.5 \mathrm{E}+07$ & $4.1 \mathrm{E}+02$ \\
\hline
\end{tabular}


Table 1. (contd)

\begin{tabular}{|c|c|c|c|c|}
\hline \multirow[b]{2}{*}{ Radionuclide } & \multirow{2}{*}{$\begin{array}{c}\text { Absorption } \\
\text { Type }\end{array}$} & \multirow{2}{*}{$\begin{array}{l}5-\mu \mathrm{m} \text { Inhalation } \\
\mathrm{e}(50) \text { in } \mathrm{Sv} \mathrm{Bq}-1\end{array}$} & \multicolumn{2}{|c|}{ Stochastic ALI } \\
\hline & & & $(\mathrm{Bq})$ & $(\mu \mathrm{Ci})$ \\
\hline Sb-126 & $\mathrm{M}$ & $3.2 \mathrm{E}-09$ & $1.6 \mathrm{E}+07$ & $4.2 \mathrm{E}+02$ \\
\hline Sb-129 & M & $3.5 \mathrm{E}-10$ & $1.4 \mathrm{E}+08$ & $3.9 \mathrm{E}+03$ \\
\hline Sc-46 & $\mathrm{S}$ & $4.8 \mathrm{E}-09$ & $1.0 \mathrm{E}+07$ & $2.8 \mathrm{E}+02$ \\
\hline Se-75 & M & 1.7E-09 & $2.9 \mathrm{E}+07$ & $7.9 \mathrm{E}+02$ \\
\hline $\mathrm{Se}-79$ & M & $3.1 \mathrm{E}-09$ & $1.6 \mathrm{E}+07$ & $4.4 \mathrm{E}+02$ \\
\hline Sm-151 & M & $2.6 \mathrm{E}-09$ & $1.9 \mathrm{E}+07$ & $5.2 \mathrm{E}+02$ \\
\hline Sn-113 & M & $1.9 \mathrm{E}-09$ & $2.6 \mathrm{E}+07$ & $7.1 \mathrm{E}+02$ \\
\hline Sn-121m & M & 3.3E-09 & $1.5 \mathrm{E}+07$ & $4.1 \mathrm{E}+02$ \\
\hline Sn-125 & M & 2.8E-09 & $1.8 \mathrm{E}+07$ & $4.8 \mathrm{E}+02$ \\
\hline Sn-126 & $\mathrm{M}$ & $1.8 \mathrm{E}-08$ & $2.8 \mathrm{E}+06$ & $7.5 \mathrm{E}+01$ \\
\hline Sr- 85 & $\mathrm{~F}$ & $5.6 \mathrm{E}-10$ & $8.9 \mathrm{E}+07$ & $2.4 \mathrm{E}+03$ \\
\hline $\mathrm{Sr}-85$ & $\mathrm{~S}$ & $6.4 \mathrm{E}-10$ & $7.8 \mathrm{E}+07$ & $2.1 \mathrm{E}+03$ \\
\hline Sr-89 & $\mathrm{F}$ & $1.4 \mathrm{E}-09$ & $3.6 \mathrm{E}+07$ & $9.7 \mathrm{E}+02$ \\
\hline Sr-89 & $\mathrm{S}$ & 5.6E-09 & $8.9 \mathrm{E}+06$ & $2.4 \mathrm{E}+02$ \\
\hline Sr-90 & $\mathrm{F}$ & $3.0 \mathrm{E}-08$ & $1.7 \mathrm{E}+06$ & $4.5 \mathrm{E}+01$ \\
\hline Sr-90 & $\mathrm{S}$ & 7.7E-08 & $6.5 \mathrm{E}+05$ & $1.8 \mathrm{E}+01$ \\
\hline Sr-91 & $\mathrm{F}$ & $2.9 \mathrm{E}-10$ & $1.7 \mathrm{E}+08$ & $4.7 \mathrm{E}+03$ \\
\hline Sr-91 & $\mathrm{S}$ & $5.7 \mathrm{E}-10$ & $8.8 \mathrm{E}+07$ & $2.4 \mathrm{E}+03$ \\
\hline Тa-182 & $\mathrm{S}$ & 7.4E-09 & $6.8 \mathrm{E}+06$ & $1.8 \mathrm{E}+02$ \\
\hline Tb-160 & $\mathrm{M}$ & 5.4E-09 & $9.3 \mathrm{E}+06$ & $2.5 \mathrm{E}+02$ \\
\hline Tc-95m & M & $8.6 \mathrm{E}-10$ & $5.8 \mathrm{E}+07$ & $1.6 \mathrm{E}+03$ \\
\hline Tc-97 & M & $1.6 \mathrm{E}-10$ & $3.1 \mathrm{E}+08$ & $8.4 \mathrm{E}+03$ \\
\hline Tc-97m & $\mathrm{M}$ & 2.7E-09 & $1.9 \mathrm{E}+07$ & $5.0 \mathrm{E}+02$ \\
\hline Tc-99 & $\mathrm{M}$ & $3.2 \mathrm{E}-09$ & $1.6 \mathrm{E}+07$ & $4.2 \mathrm{E}+02$ \\
\hline Tc-99m & $\mathrm{M}$ & $2.9 \mathrm{E}-11$ & $1.7 \mathrm{E}+09$ & $4.7 \mathrm{E}+04$ \\
\hline Te-123m & $\mathrm{M}$ & 3.4E-09 & $1.5 \mathrm{E}+07$ & $4.0 \mathrm{E}+02$ \\
\hline Te-125m & $\mathrm{M}$ & 2.9E-09 & $1.7 \mathrm{E}+07$ & $4.7 \mathrm{E}+02$ \\
\hline Te-132 & Vapor & 5.1E-09 & $9.8 \mathrm{E}+06$ & $2.6 \mathrm{E}+02$ \\
\hline Th-227 & M & $6.2 \mathrm{E}-06$ & $8.1 \mathrm{E}+03$ & $2.2 \mathrm{E}-01$ \\
\hline Th-227 & $\mathrm{S}$ & 7.6E-06 & $6.6 \mathrm{E}+03$ & $1.8 \mathrm{E}-01$ \\
\hline Th-228 & M & $2.2 \mathrm{E}-05$ & $2.3 \mathrm{E}+03$ & $6.1 \mathrm{E}-02$ \\
\hline Th-228 & $\mathrm{S}$ & $2.5 \mathrm{E}-05$ & $2.0 \mathrm{E}+03$ & $5.4 \mathrm{E}-02$ \\
\hline Th-229 & M & $6.9 \mathrm{E}-05$ & $7.2 \mathrm{E}+02$ & $2.0 \mathrm{E}-02$ \\
\hline Th-229 & $\mathrm{S}$ & $4.8 \mathrm{E}-05$ & $1.0 \mathrm{E}+03$ & $2.8 \mathrm{E}-02$ \\
\hline Th-230 & $\mathrm{M}$ & $2.8 \mathrm{E}-05$ & $1.8 \mathrm{E}+03$ & $4.8 \mathrm{E}-02$ \\
\hline Th-230 & $\mathrm{S}$ & $7.2 \mathrm{E}-06$ & $6.9 \mathrm{E}+03$ & $1.9 \mathrm{E}-01$ \\
\hline Th-232 & $\mathrm{M}$ & $2.9 \mathrm{E}-05$ & $1.7 \mathrm{E}+03$ & 4.7E-02 \\
\hline Th-232 & $\mathrm{S}$ & $1.2 \mathrm{E}-05$ & $4.2 \mathrm{E}+03$ & $1.1 \mathrm{E}-01$ \\
\hline Th-234 & M & 5.3E-09 & $9.4 \mathrm{E}+06$ & $2.5 \mathrm{E}+02$ \\
\hline Th-234 & $\mathrm{S}$ & $5.8 \mathrm{E}-09$ & $8.6 \mathrm{E}+06$ & $2.3 \mathrm{E}+02$ \\
\hline Tl-204 & $\mathrm{F}$ & $6.2 \mathrm{E}-10$ & $8.1 \mathrm{E}+07$ & $2.2 \mathrm{E}+03$ \\
\hline Tm-171 & $\mathrm{M}$ & $9.1 \mathrm{E}-10$ & $5.5 \mathrm{E}+07$ & $1.5 \mathrm{E}+03$ \\
\hline U-232 & $\mathrm{M}$ & $4.8 \mathrm{E}-06$ & $1.0 \mathrm{E}+04$ & $2.8 \mathrm{E}-01$ \\
\hline U-232 & $\mathrm{S}$ & $2.6 \mathrm{E}-05$ & $1.9 \mathrm{E}+03$ & $5.2 \mathrm{E}-02$ \\
\hline U-233 & $\mathrm{M}$ & $2.2 \mathrm{E}-06$ & $2.3 \mathrm{E}+04$ & $6.1 \mathrm{E}-01$ \\
\hline U-233 & $\mathrm{S}$ & $6.9 \mathrm{E}-06$ & $7.2 \mathrm{E}+03$ & $2.0 \mathrm{E}-01$ \\
\hline U-234 & M & $2.1 \mathrm{E}-06$ & $2.4 \mathrm{E}+04$ & $6.4 \mathrm{E}-01$ \\
\hline U-234 & $\mathrm{S}$ & $6.8 \mathrm{E}-06$ & $7.4 \mathrm{E}+03$ & $2.0 \mathrm{E}-01$ \\
\hline
\end{tabular}


Table 1. (contd)

\begin{tabular}{|c|c|c|c|c|}
\hline \multirow[b]{2}{*}{ Radionuclide } & \multirow{2}{*}{$\begin{array}{c}\text { Absorption } \\
\text { Type }\end{array}$} & \multirow{2}{*}{$\begin{array}{l}5-\mu m \text { Inhalation } \\
\mathrm{e}(50) \text { in } \mathrm{Sv} \mathrm{Bq}-1\end{array}$} & \multicolumn{2}{|c|}{ Stochastic ALI } \\
\hline & & & $(\mathrm{Bq})$ & $(\mu \mathrm{Ci})$ \\
\hline U-235 & $\mathrm{M}$ & $1.8 \mathrm{E}-06$ & $2.8 \mathrm{E}+04$ & $7.5 \mathrm{E}-01$ \\
\hline U-235 & $\mathrm{S}$ & $6.1 \mathrm{E}-06$ & $8.2 \mathrm{E}+03$ & $2.2 \mathrm{E}-01$ \\
\hline U-236 & $\mathrm{M}$ & $1.9 \mathrm{E}-06$ & $2.6 \mathrm{E}+04$ & $7.1 \mathrm{E}-01$ \\
\hline U-236 & $\mathrm{S}$ & $6.3 \mathrm{E}-06$ & $7.9 \mathrm{E}+03$ & $2.1 \mathrm{E}-01$ \\
\hline U-238 & M & $1.6 \mathrm{E}-06$ & $3.1 \mathrm{E}+04$ & $8.4 \mathrm{E}-01$ \\
\hline U-238 & $\mathrm{S}$ & 5.7E-06 & $8.8 \mathrm{E}+03$ & $2.4 \mathrm{E}-01$ \\
\hline U-239 & M & $3.3 \mathrm{E}-11$ & $1.5 \mathrm{E}+09$ & $4.1 \mathrm{E}+04$ \\
\hline U-239 & $\mathrm{S}$ & $3.5 \mathrm{E}-11$ & $1.4 \mathrm{E}+09$ & $3.9 \mathrm{E}+04$ \\
\hline$V-49$ & $\mathrm{~F}$ & $2.6 \mathrm{E}-11$ & $1.9 \mathrm{E}+09$ & $5.2 \mathrm{E}+04$ \\
\hline W-185 & $\mathrm{F}$ & $2.2 \mathrm{E}-10$ & $2.3 \mathrm{E}+08$ & $6.1 \mathrm{E}+03$ \\
\hline W-187 & $\mathrm{F}$ & $3.3 \mathrm{E}-10$ & $1.5 \mathrm{E}+08$ & $4.1 \mathrm{E}+03$ \\
\hline Y-88 & M & 3.3E-09 & $1.5 \mathrm{E}+07$ & $4.1 \mathrm{E}+02$ \\
\hline$Y-88$ & $\mathrm{~S}$ & $3.0 \mathrm{E}-09$ & $1.7 \mathrm{E}+07$ & $4.5 \mathrm{E}+02$ \\
\hline$Y-90$ & M & $1.6 \mathrm{E}-09$ & $3.1 \mathrm{E}+07$ & $8.4 \mathrm{E}+02$ \\
\hline$Y-90$ & $\mathrm{~S}$ & 1.7E-09 & $2.9 \mathrm{E}+07$ & $7.9 \mathrm{E}+02$ \\
\hline $\mathrm{Zn}-65$ & $\mathrm{~S}$ & 2.8E-09 & $1.8 \mathrm{E}+07$ & $4.8 \mathrm{E}+02$ \\
\hline Zr-93 & $\mathrm{F}$ & $2.9 \mathrm{E}-08$ & $1.7 \mathrm{E}+06$ & $4.7 \mathrm{E}+01$ \\
\hline $\mathrm{Zr}-95$ & S & 4.2E-09 & $1.2 \mathrm{E}+07$ & $3.2 \mathrm{E}+02$ \\
\hline
\end{tabular}

\subsection{References}

10 CFR 835. 2009. U.S. Department of Energy, Occupational Radiation Protection. U.S. Code of Federal Regulations. Accessed on 7/28/2009 at http://www.gpoaccess.gov/cfr.

International Commission on Radiological Protection (ICRP). 1979. Limits for Intakes of Radionuclides by Workers. ICRP Publication 30 Part 1. Annals of the ICRP, Volume 2 No. 3/4. Pergamon Press.

International Commission on Radiological Protection (ICRP). 1991. Annual Limits on Intake of Radionuclides by Workers Based on the 1990 Recommendations. Annals of the ICRP, Volume 21 No 4. Pergamon Press.

International Commission on Radiological Protection (ICRP). 1998. The ICRP Database of Dose Coefficients: Workers and Members of the Public. Version 2.01, update 2001. Pergamon. Distributed by Elsevier Science Ltd., Tarrytown, NY.

U.S. Environmental Protection Agency. 1988. Limiting Values of Radionuclide Intake and Air Concentration and Dose Conversion Factors for Inhalation, Submersion, and Ingestion. Federal Guidance Report No. 11. U.S. Environmental Protection Agency. Washington, D.C.

U.S. Department of Energy. 2004. DOE Handbook - Radiological Control Programs for Special Tritium Compounds. DOE-HBK-1184-2004. U.S. Department of Energy. Washington, D.C. 